\title{
Invariant Sets for a Class of Hybrid Systems
}

\author{
Mats Jirstrand \\ Department of Electrical Engineering \\ Linköping University, S-581 83 Linköping, Sweden \\ WWW: http://www.control.isy.liu.se \\ Email: matsj@isy.liu.se
}

March 4, 1998

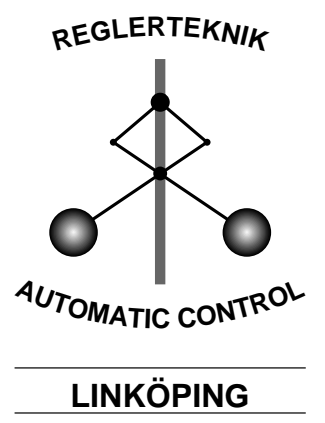

Report no.: LiTH-ISY-R-2025

Submitted to CDC98

Technical reports from the Automatic Control group in Linköping are available by anonymous ftp at the address ftp.control.isy.liu.se. This report is contained in the compressed postscript file 2025.ps.Z. 



\title{
Invariant Sets for a Class of Hybrid Systems
}

\author{
Mats Jirstrand \\ Department of Electrical Engineering, \\ Linköping University, 58183 Linköping, Sweden \\ www: www.control.isy.liu.se \\ email: matsj@isy.liu.se
}

March 4, 1998

\begin{abstract}
A method for computing invariant sets of piecewise affine systems is presented. The method is based on convex optimization techniques and linear matrix inequalities. We show how to compute invariant ellipsoids, quadratic cones, and paraboloids for affine systems which contain initial sets of different form such as polytopes, ellipsoids, and degenerate ellipsoids. An invariant set for a piecewise affine system can be computed by an iterative procedure that utilizes these types of computations.
\end{abstract}

\section{Introduction}

Invariant sets of dynamic systems play an important role in many situations when the dynamic behavior is constrained in some way. Knowing that a set in the state space of a system is invariant means that we have bounds on the behavior. We can verify that prespecified bounds which originates from e.g., safety restrictions, physical constraints, or state-feedback magnitude bounds are not invalidated. This verification becomes subset inclusion tests, i.e., we have to check if the invariant set is a subset of the admissible part of the state space.

For piecewise affine systems, i.e., systems whose dynamics is given by an affine differential equation in different polyhedral regions of the state space, the computation of invariant sets is harder than for systems defined by state equations with continuous right hand sides since both continuous and discrete dynamics have to be taken into account. This kind of systems appear 
e.g., when a linear system is controlled by a PID-controller equipped with selectors [2], if there are saturations present in the system, or as approximations of nonlinear systems obtained by system identification using hinging hyperplanes [5].

Controller design and verification based on invariant sets has been studied in $[7,16,8,9,17,15,18]$. However, the continuous dynamics in the studied systems are either of very simple form (constant right hand sides or rectangular differential inclusions) or if more complex dynamics are considered the ability to use constructive methods are lost. This contribution is an attempt to allow verification of hybrid systems with more advanced continuous dynamics without sacrificing the constructive part.

The paper is organized as follows. In Section 2 we give a definition of piecewise affine systems. Section 3 treats invariant sets for continuous systems. A number of results for invariant quadratic sets such as ellipsoids, quadratic cones, and paraboloids are given here. In Section 4 we use the results on quadratic invariant sets to compute invariant sets of piecewise affine systems. Two examples illustrating the results are presented in Section 5 and a summary is given in Section 6 .

\section{Piecewise Affine Systems}

By a piecewise affine system we mean a dynamic system which has different affine dynamics in different parts of the continuous state space.

To describe the state of the system we require two distinct sets of states. The set of continuous states $x \in \mathbb{R}^{n}$ and the set of discrete states (or equivalently modes $) s \in S=\{1, \ldots, N\}$. For each discrete state the continuous state evolves according to an affine differential equation

$$
\dot{x}(t)=A_{s(x(t))} x(t)+b_{s(x(t))},
$$

where $A_{s(x(t))} \in \mathbb{R}^{n \times n}, b_{s(x(t))} \in \mathbb{R}^{n}$ and $s: \mathbb{R}^{n} \rightarrow S$. The function $s$ is determined by a number of transition sets, see below. We may think of the state space of (1) as a collection of $N$ enumerated copies of $\mathbb{R}^{n}$. A simple generalization is to let the continuous part of the state space have variable dimension, i.e., $x \in \mathbb{R}^{n_{s(t)}}$. This is needed in some applications.

The discrete and continuous dynamics is coupled through a number of transition sets $T_{i j} \in \mathbb{R}^{n}$. We use the convention that $T_{i i}=\emptyset$. A discrete state transition from mode $i$ to mode $j$ occurs when the continuous state hits the boundary of a transition set, i.e., $x(t) \in \partial T_{i j}$. Then the continuous state is mapped into the continuous state space of mode $j$. For simplicity we 
let this mapping be the identity mapping. We assume that each transition set is defined by a halfspace

$$
T_{i j}=\left\{x \in \mathbb{R}^{n} \mid c_{i j}^{T} x \leq d_{i j}\right\}
$$

A piecewise affine system can be thought of as a finite state machine with a continuous system at each discrete state, see Figure 1. In $[1,6,15]$ the term hybrid automaton are used for system models similar to the ones studied here. Stability is related to invariant sets since it is a sufficient

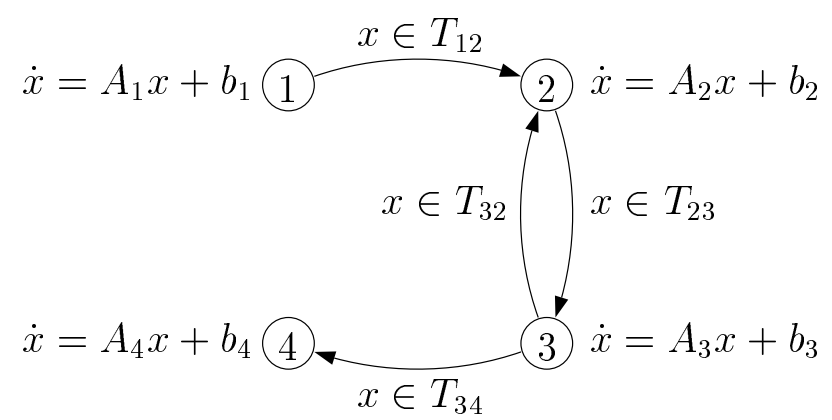

Figure 1: A hybrid automaton with four modes.

condition for invariance. Stability of piecewise linear and affine systems has been investigated in $[12,11,4,14]$.

\section{Invariant Sets}

An invariant set of a dynamic system is a subset of the state space such that once the state enters this set it will remain in it for all future times, i.e.,

$$
z\left(t_{1}\right) \in M \Longrightarrow z(t) \in M \text { for all } t>t_{1},
$$

where $z(t)$ is the state of the dynamic system at time $t$ and $M$ is a subset of the state space. Sometimes the term positive invariant set is used to stress that the invariance only holds for future times and not backwards in time [13].

Consider the following dynamic system

$$
\dot{x}=f(x),
$$

where $f: \mathbb{R}^{n} \rightarrow \mathbb{R}^{n}$ is a continuously differentiable function. A necessary and sufficient condition for invariance of a set $M$ is that the flow of the differential 
equation is directed into the set at each point on the boundary, $\partial M$. For a set with smooth boundary this can be expressed as follows

$$
n_{M}(x)^{T} f(x) \leq 0 \quad \forall x \in \partial M,
$$

where $n_{M}(x)$ denotes a normal to $\partial M$ at $x$. If the invariant set is described by an inequality

$$
M=\left\{x \in \mathbb{R}^{n} \mid V(x) \leq c\right\},
$$

i.e., as a level set of a continuously differentiable function $V: \mathbb{R}^{n} \rightarrow \mathbb{R}$, a normal is given by the gradient $V_{x}$ of $V$. In this case condition (4) becomes

$$
V_{x}(x) f(x) \leq 0 \quad \forall x \quad \text { such that } \quad V(x)=c .
$$

If $V(\theta, x)$ is a parameterized class of functions, inequality (6) can be used to derive restrictions on the parameters, $\theta$ such that $V(\theta, x) \leq c$ describes an invariant set.

Observe that level sets of Lyapunov functions of dynamic systems are invariant sets since the flow of the vector field is always directed into the set according to the definition of a Lyapunov function.

It is easy to show that invariance of a set w.r.t. a dynamic system is a property not affected by a diffeomorphism, see [10].

\subsection{Affine Systems}

For linear (or affine) systems there are some particularly simple types of invariant sets which are closely related to the eigenvalues and eigenvectors of the system matrix. These are invariant sets described by linear and quadratic inequalities such as invariant halfspaces, slabs, polyhedra, ellipsoids, cones, and paraboloids.

The affine system $\dot{x}=A x+b$ can always be transformed to a linear system by the coordinate change $z=x+A^{-1} b$ if $A$ is nonsingular. In the sequel we will assume that all systems have nonsingular system matrices.

Observe that if $A \in \mathbb{R}^{n \times n}$ is nonsingular and has a real eigenvalue $\lambda \in \mathbb{R}$ then we can rewrite $\dot{x}=A x+b$ as

$$
\dot{z}=\left[\begin{array}{ll}
\tilde{A} & 0 \\
0 & \lambda
\end{array}\right] z .
$$

Hence, we can w.l.o.g. consider only linear systems. 


\subsection{Convex Optimization and Quadratic Sets}

We will formulate the computation of invariant sets as convex optimization problems of the following form

$$
\begin{array}{ll}
\text { minimize } & \log \operatorname{det} P^{-1} \\
\text { subject to } & P \succ 0, \\
& \operatorname{LMI}(P, q),
\end{array}
$$

where $P^{T}=P \in \mathbb{R}^{n \times n}$ is a matrix variable and $P \succ 0$ means that $P$ is positive definite. $\operatorname{LMI}(P, q)$ denotes one or several linear matrix inequalities (LMI:s) in $P$ and some additional variables $q$. This optimization problem is convex [3] and can be solved very efficiently [19]. In our case the objective corresponds to minimization of an ellipsoidal volume and the LMI:s correspond to various constraints such as invariance w.r.t. to a dynamic system and inclusion of initial sets in the invariant set.

Many operations on quadratic and affine sets, such as intersections, projections, test of emptiness, etc. can be carried out in a common framework [10]. All operations needed in the methods proposed in this paper are implemented as a package in MATHEMATICA. We also use SDPSOL which is a solver for semidefinite programming and determinant maximization problems, see [20, 21].

\subsection{Invariant Ellipsoids}

Every asymptotically stable linear or affine system has ellipsoidal invariant sets. This follows from the existence of a quadratic Lyapunov function for such systems and that levelsets of Lyapunov functions are invariant sets. We summarize

Lemma 1 Let $\dot{x}=A x, x \in \mathbb{R}^{n}, A \in \mathbb{R}^{n \times n}$, where all eigenvalues of $A$ lies in the open left halfplane. Then there exist invariant ellipsoids.

Given a set of initial states, we are interested in computing the smallest invariant ellipsoid that contains these states. For a number of initial sets such as a point, a convex hull of points, and ellipsoids this is a convex optimization problem, see [3].

Suppose that we have the following representation of an ellipsoid

$$
\mathcal{E}=\left\{x \in \mathbb{R}^{n} \mid\left(x-x_{c}\right)^{T} P\left(x-x_{c}\right) \leq 1\right\},
$$

where $P^{T}=P \succ 0$ and $x_{c} \in \mathbb{R}^{n}$ is the center. Then its volume can be measured by the function $\log \operatorname{det} P^{-1}$, see [3]. 
Let the set of initial states be the polytope (or convex hull of points) $\operatorname{Co}\left\{v_{1}, \ldots, v_{p}\right\}$. Then the minimal volume invariant ellipsoid w.r.t. $\dot{x}=A x$ which is centered at the origin and contains this set can be computed by

$$
\begin{array}{ll}
\operatorname{minimize} & \log \operatorname{det} P^{-1} \\
\text { subject to } & A^{T} P+P A \preceq 0, \quad P \succ 0, \\
& v_{i}^{T} P v_{i} \leq 1, \quad i=1, \ldots, p
\end{array}
$$

which is of the form (8). The last $p$ inequalities ensure that the vertices of the polytope belongs to $\mathcal{E}$. Since ellipsoids are convex sets it follows that $\operatorname{Co}\left\{v_{1}, \ldots, v_{p}\right\} \subseteq \mathcal{E}$.

The condition $\mathcal{E}_{0} \subseteq \mathcal{E}$ can be formulated as a linear matrix inequality using the so called S-procedure [3], which can be used to ensure that a quadratic function is negative whenever another quadratic function is negative. It can be shown that the inclusions is true if and only if there exists a $\tau \geq 0$ such that

$$
\left[\begin{array}{cc}
P & 0 \\
0 & -1
\end{array}\right]-\tau\left[\begin{array}{cc}
P_{0} & -P_{0} x_{0} \\
-x_{0}^{T} P_{0} & x_{0}^{T} P_{0} x_{0}-1
\end{array}\right] \preceq 0
$$

which is a linear matrix inequality in $P$ and $\tau$. Substituting the last $p$ inequalities in (9) we get a convex optimization problem for computing the smallest invariant ellipsoid that contains $\mathcal{E}_{0}$. An LMI for describing that the intersection of an ellipsoid and an affine set $\left\{x \in \mathbb{R}^{n} \mid C x=d\right\}$ is contained in an ellipsoid is only a slight variation of (10)

$$
\left[\begin{array}{cc}
C_{\mathcal{N}} & C^{\dagger} d \\
0 & 1
\end{array}\right]^{T} Q(\tau)\left[\begin{array}{cc}
C_{\mathcal{N}} & C^{\dagger} d \\
0 & 1
\end{array}\right] \preceq 0,
$$

where $Q(\tau)$ is the left hand side of the LMI in (10), $C^{\dagger}$ is the pseudo inverse of $C$, and the columns of $C_{\mathcal{N}}$ is a basis of the nullspace of $C$. Geometrically, this corresponds to a projection of constraint (10) onto the affine set.

\subsection{Invariant Quadratic Cones and Paraboloids}

A left eigenvector corresponding to a real eigenvalue of a system matrix has interesting geometrical interpretations. We can always decouple a system in two parts according to (7). Under some additional constraints this observation can be used to show that there are invariant sets whose intersection with hyperplanes orthogonal to a left eigenvector are ellipsoids. Both quadratic cones and paraboloids have this property. Let $x=\left(\tilde{x}, x_{n}\right)^{T} \in \mathbb{R}^{n}$. A quadratic cone can be described as

$$
\mathcal{Q C}=\left\{x \in \mathbb{R}^{n} \mid \tilde{x}^{T} P \tilde{x} \leq x_{n}^{2}\right\},
$$


and a paraboloid by

$$
\mathcal{P}=\left\{x \in \mathbb{R}^{n} \mid \tilde{x}^{T} P \tilde{x} \leq \pm x_{n}\right\}
$$

In both (12) and (13) we have $P^{T}=P \succ 0$.

Now, consider the function $V(x)=\tilde{x}^{T} P \tilde{x}-x_{n}^{\gamma}$, where $P^{T}=P \in$ $\mathbb{R}^{(n-1) \times(n-1)}$ with $P \succ 0$ and $\gamma>0$.

Lemma 2 The set $\left\{x \in \mathbb{R}^{n} \mid V(x) \leq 0\right\}$ is invariant w.r.t. (7) if and only if

$$
\tilde{A}^{T} P+P \tilde{A}-\gamma \lambda P \preceq 0 .
$$

Proof: Using inequality (6) for the above class of functions we get

$$
\begin{aligned}
& {\left[\begin{array}{ll}
2 \tilde{x}^{T} P & -\gamma x_{n}^{\gamma-1}
\end{array}\right]\left[\begin{array}{c}
\tilde{A} \tilde{x} \\
\lambda x_{n}
\end{array}\right]=} \\
& \\
& \quad \tilde{x}^{T}\left(\tilde{A}^{T} P+P \tilde{A}\right) \tilde{x}-\gamma \lambda x_{n}^{\gamma} \leq 0,
\end{aligned}
$$

where the equality follows by symmetrization. This inequality has to be satisfied for all $x$ on the boundary of the set, i.e., for $x$ satisfying $x_{n}^{\gamma}=\tilde{x}^{T} P \tilde{x}$. With this substitution (14) becomes

$$
\tilde{x}^{T}\left(\tilde{A}^{T} P+P \tilde{A}-\gamma \lambda P\right) \tilde{x} \leq 0, \quad \forall \tilde{x}
$$

and the lemma follows.

Observe that we do not require system (7) to be stable in the above lemma. It can be shown that the LMI in Lemma 2 has a solution if and only if $\operatorname{Re}[\tilde{\lambda}] \leq \gamma \lambda / 2$ where $\tilde{\lambda}$ denotes any eigenvalue of $\tilde{A}$. Note that for $0<\gamma<1$ the level set is not convex. Now, $\gamma=1$ and $\gamma=2$ give invariant quadratic cones and paraboloids, respectively.

Corollary 1 Let $\dot{x}=A x, x \in \mathbb{R}^{n}, A \in \mathbb{R}^{n \times n}$. If the eigenvalue of $A$ with maximal real part $\lambda$ is real. Then there exist invariant quadratic cones.

Corollary 2 Let $\dot{x}=A x, x \in \mathbb{R}^{n}, A \in \mathbb{R}^{n \times n}$. If there exists a real eigenvalue $\lambda$ of $A$ such that $\operatorname{Re}[\tilde{\lambda}] \leq \lambda / 2$, where $\tilde{\lambda}$ denotes any other eigenvalue of A. Then there exist invariant paraboloids.

Similar to the computation of invariant ellipsoids, we are interested in computing invariant quadratic cones and paraboloids that contain a given set of initial states. To measure the size of a quadratic cone or paraboloid 
we use the volume of the ellipsoid given by the intersection of the quadratic cone (12) or paraboloid (13) and a hyperplane $x_{n}=1$.

Let $\mathbf{C o}\left\{v_{1}, \ldots, v_{p}\right\}$ be a polytope of initial states such that $\left(v_{i}\right)_{n}, i=$ $1, \ldots, p$ all have the same sign. Here $\left(v_{i}\right)_{n}$ denotes the last component of $v_{i}$. If this assumption is invalid then there are no invariant quadratic cones or paraboloids for this initial polytope. The computation of an invariant quadratic cone can be formulated as a convex optimization problem according to

$$
\begin{array}{ll}
\operatorname{minimize} & \log \operatorname{det} P^{-1} \\
\text { subject to } & \tilde{A}^{T} P+P \tilde{A}-2 \lambda P \prec 0, \quad P \succ 0, \\
& \tilde{v}_{i}^{T} P \tilde{v}_{i}<\left(v_{i}\right)_{n}^{2}, \quad i=1, \ldots, p .
\end{array}
$$

For an invariant paraboloid we get

$$
\begin{array}{ll}
\operatorname{minimize} & \log \operatorname{det} P^{-1} \\
\text { subject to } & \tilde{A}^{T} P+P \tilde{A}-\lambda P \prec 0, \quad P \succ 0, \\
& \tilde{v}_{i}^{T} P \tilde{v}_{i}< \pm\left(v_{i}\right)_{n}, \quad i=1, \ldots, p .
\end{array}
$$

Using the so called S-procedure $\mathcal{E}_{0} \subseteq \mathcal{Q C}$ can be formulated as a linear matrix inequality

$$
\left[\begin{array}{ccc}
P & 0 & 0 \\
0 & -1 & 0 \\
0 & 0 & 0
\end{array}\right]-\tau\left[\begin{array}{cc}
P_{0} & -P_{0} x_{0} \\
-x_{0}^{T} P_{0} & x_{0}^{T} P_{0} x_{0}-1
\end{array}\right] \preceq 0
$$

and the inclusion $\mathcal{E}_{0} \subseteq \mathcal{P}$ can be obtained using

$$
\left[\begin{array}{ccc}
P & 0 & 0 \\
0 & 0 & \mp 1 / 2 \\
0 & \mp 1 / 2 & 0
\end{array}\right]-\tau\left[\begin{array}{cc}
P_{0} & -P_{0} x_{0} \\
-x_{0}^{T} P_{0} & x_{0}^{T} P_{0} x_{0}-1
\end{array}\right] \preceq 0
$$

In both (17) and (18) $\tau \geq 0$ and $x_{0}$ is the center of $\mathcal{E}_{0}$. Inclusion of an intersection of an ellipsoid, quadratic cone or paraboloid and an affine set can be formulated similar to (11). Using (17) or (18) instead of the last $p$ inequalities in (15) or (16) we can compute invariant quadratic cones or paraboloids that contains $\mathcal{E}_{0}$.

Note that the initial set has to belong to either $x_{n}>0$ or $x_{n}<0$ for an invariant quadratic cone or paraboloid to exist. If this is the case the \pm sign is determined by checking on which side of $x_{n}=0$ the initial set is located. 


\subsection{Piecewise Affine Systems}

For a piecewise affine system an invariant set is a subset of $\mathbb{R}^{n} \times\{1, \ldots, N\}$, i.e., an collection of sets in $M_{i} \subseteq \mathbb{R}^{n}, i=1, \ldots, N$,

$$
\left\{(x, s) \mid x \in M_{s}, s \in\{1, \ldots, N\}\right\} .
$$

We will call the sets $M_{i}, i=1, \ldots, N$ the mode invariant sets of system (1). Observe that a mode invariant set $M_{i}$ does not have to be connected due to the switching behavior of the system. Furthermore, inequality (4) does not have to be valid on the boundary of $M_{i}$. However, either inequality (4) is valid at a point $x \in \partial M_{i}$ or $x \in T_{i j}$, where $T_{i j}$ is the switching set defining transitions between the discrete states $i$ and $j$. Furthermore, the definition of a piecewise affine system implies that if $x(t-) \in \partial M_{i} \cap T_{i j}$ then $x(t) \in M_{j}$, where $t$ is the switching time. The set $\partial M_{i} \cap T_{i j}$ is contained in a mode invariant set of mode $j$. To ensure such inclusions in computations, conditions such as (11) are useful.

Note that if a component of a mode invariant set does not intersect any of the transition sets of the mode, the state will remain in this component for all future times.

\section{Invariant Sets for Piecewise Affine Systems}

The computations of invariant sets for linear systems presented in the previous section can be used for computations of invariant sets of piecewise affine systems.

The Problem Suppose that we have an initial set of states $\mathcal{X} \mathcal{S}_{0} \subseteq \mathbb{R}^{N} \times$ $\{1, \ldots, N\}$ of a piecewise affine system such that for all $(x, s) \in \mathcal{X} \mathcal{S}_{0}$ we have $s \equiv i \in\{1, \ldots, N\}$. Furthermore, let $\mathcal{X}_{0}=\left\{x \mid(x, s) \in \mathcal{X S}_{0}\right\}$. In other words we have an initial set of continuous states $\mathcal{X}_{0}$ in mode $i$. We now want to compute an invariant set for a piecewise affine system which contains this set of initial states. Using the notion for mode invariant sets introduced in the previous section, we have the condition

$$
\mathcal{X} \mathcal{S}_{0} \subseteq\left\{(x, s) \mid x \in M_{s}, s \in\{1, \ldots, N\}\right\} .
$$

A Computational Procedure We will now describe an iterative procedure for computing an invariant set of a piecewise affine system. We utilize an abstraction of the piecewise affine system for computing invariant sets. Instead of considering specific trajectories in the continuous state space of each 
mode we focus on invariant sets w.r.t. the affine dynamics in the mode. The evolution of the abstracted system can be described by a nondeterministic finite state machine, which means that several transitions are possible from each mode simultaneously. These transitions are determined by checking if the currently computed invariant set of the mode intersects the transition sets.

We first give a brief description of the computational procedure and then formalize the steps as an iterative application of an update function on a set of mode objects.

Given a set of initial continuous states in a mode we compute an invariant set that contains this initial set. This is in fact an overapproximation of the set of reachable states for these initial states. We can then compute the intersection of the invariant set and the transition sets of the mode which gives an overapproximation of the continuous states that will be mapped into other modes by the transition mechanism. These mapped sets becomes initial sets for new invariant set computations and the procedure is repeated. The procedure terminates if there are no new nonempty intersections with transition sets or if the intersections are mapped into already computed invariant sets.

The computations performed in each step of the iterative procedure can be described as a function, the update function operating on a finite set of mode objects $\left\{\operatorname{MODE}_{i}\right\}_{i=1}^{N}$ which correspond to the modes or discrete states of the piecewise affine system.

To formulate the procedure we need some additional notation. Let $M_{i}^{k} \subseteq$ $\mathbb{R}^{n}$ denote a mode invariant set component of mode $i$. A mode invariant set component is an invariant set w.r.t. to the affine dynamics in the mode if we we neglect the transition mechanism. The following relation between the mode invariant set and mode invariant set component holds

$$
M_{i}=\bigcup_{k=1}^{\infty} M_{i}^{k} \bigcap_{j=1}^{N} \overline{T_{i j}^{c}},
$$

where $\overline{T_{i j}^{c}}$ denotes the closure of the complement of the transition set $T_{i j}$. The infinite union of mode invariant set components is in fact finite if the iterative procedure terminates.

A mode initial set, $I_{i} \subseteq \mathbb{R}^{n}$, is a set of continuous states in mode $i$ which is used as input to a computation of a mode invariant set component. Examples of mode initial sets are the initial set of continuous states $\mathcal{X}_{0}$ but also sets of continuous states that enters a mode due to the transition mechanism. Suppose that $M_{i}^{k} \cap T_{i j}$ is nonempty. According to the transition mechanism the states in this set is mapped into the continuous state space of mode $j$. This set will be a mode initial set in mode $j$ in the next iteration. Note that, 
at each iteration, a new mode initial set is produced in a mode if a currently computed mode invariant set component intersects some transition set.

The Mode Object The mode object is a data structure for storing information about a mode of a piecewise affine system such as the continuous dynamics, the transition sets, the mode invariant set components, and the mode initial sets. The structure of the mode object are

$$
\operatorname{ModE}_{i}= \begin{cases}\dot{x}=A_{i} x+b_{i} & \text { Affine dynamics } \\
\left\{T_{i 1}, \ldots, T_{i N}\right\} & \text { List of transition sets } \\
\left\{M_{i}^{1}, \ldots, M_{i}^{l}\right\} & \text { List of mode invariant } \\
& \text { set components } \\
\left\{I_{i}^{1}, \ldots, I_{i}^{m}\right\} & \begin{array}{l}
\text { Current list of mode } \\
\text { initial sets }
\end{array}\end{cases}
$$

Initially only one mode object has a nonempty mode initial set, $\mathcal{X}_{0}$. The iterative procedure terminates when all lists of mode initial sets in the mode objects are empty.

The Update Function The update function, which we denote $\mathcal{F}$, operates on the current set of mode objects and produce an updated version of it. We have

$$
\mathcal{F}:\left\{\operatorname{MoDE}_{i}\right\}_{i=1}^{N} \rightarrow\left\{\operatorname{MODE}_{i}\right\}_{i=1}^{N}
$$

Termination of the iterative procedure corresponds to a fixpoint of the update function. Let $\left\{\operatorname{MoDE}_{i}\right\}_{i=1}^{N}$ denote the current set of mode objects and $\left\{\operatorname{MoDE}_{i}^{\prime}\right\}_{i=1}^{N}$ the updated set of mode objects. When the update function is applied to the current set of mode objects the new set of mode objects are computed as follows. First, every mode object $\operatorname{MODE}_{i}^{\prime}$ is given the same value as $\operatorname{MODE}_{i}$ but with an empty list of mode initial sets. For each mode object $\mathrm{MODE}_{i}$ with a nonempty list of mode initial sets we proceed as follows.

1. Decide if every mode initial set belongs to some of the mode invariant set components, i.e., check if $I_{i}^{p} \subseteq M_{i}^{k}, p=1, \ldots, m, k=1, \ldots, l$. Let $P$ be the set of values of $p$ for which this is not the case. If $P=\emptyset$ then skip rest of the items in this list.

2. Compute a mode invariant set, $M_{i}^{k^{\prime}}$ such that $I_{i}^{p} \subseteq M_{i}^{k^{\prime}}, p \in P$.

3. Append $M_{i}^{k^{\prime}}$ to the list of mode invariant set components of $\mathrm{MODE}_{i}^{\prime}$. 
4. Decide if $M_{i}^{k^{\prime}} \cap T_{i j}, j=1, \ldots, N$ is empty (trivial if $T_{i j}=\emptyset$ ). Let $J$ denote the set of values of $j$ which correspond to nonempty intersections.

5. Append $M_{i}^{k^{\prime}} \cap T_{i j}$ to the list of mode initial sets of $\operatorname{MoDE}_{j}^{\prime}$ for $j \in J$.

The last item in the list corresponds to the mapping of the continuous states from mode $i$ to mode $j$ due to the transition mechanism.

The number of mode invariant set components in the list of each mode object may not grow during an iteration by two reasons. First, there are no mode initial sets. This means that no transitions to this mode was detected in the previous iteration and we do not have to compute a mode invariant set component.

Second, the mode initial sets already belong to the elements of the list of mode invariant set components. This corresponds to that all the continuous states, that are mapped from other modes by the transition mechanism in the previous iteration, enter subsets of the continuous state space of this mode whose future behavior is already approximated. This is an indication of that some kind of cyclic behavior between the discrete states can occur. However, since the computation of invariant sets is only an overapproximation of the set of reachable states this might not correspond to a cyclic behavior.

The intersection between a mode invariant set component and the transition sets are overapproximations of the sets of continuous states that will be mapped into new modes. Hence, it is important to keep the size of these intersections as small as possible. For stable systems which have both invariant paraboloids and quadratic cones one should choose invariant quadratic cones. This will give a smaller intersection between the invariant set and any hyperplane that separates the initial set and the stationary point (i.e., the vertex of the cone or paraboloid). If the system is unstable we should choose a paraboloid if possible instead of a quadratic cone.

\section{$5 \quad$ Examples}

Here we will give two examples where we compute invariant sets for piecewise affine systems. We choose continuous dynamics in $\mathbb{R}^{3}$ since trajectories and sets then are easy to visualize. However, this is only for clarity of presentation. Convex optimization computations, which the computational procedures are based on, are very efficient in practise [3] and is scalable to much larger problems. 
Example 1 Consider the hybrid automaton shown in Figure 1. Suppose that mode 4 corresponds to a forbidden mode due to some safety or performance reasons. The aim of this example is to show that given a specific set of initial states we will never enter the forbidden state.

Let the transition sets and affine systems be given by

$$
\begin{aligned}
& T_{12}=\left\{x \in \mathbb{R}^{3} \mid x_{1}+x_{2}+x_{3} \geq 9.6\right\} \\
& T_{23}=\left\{x \in \mathbb{R}^{3} \mid x_{1} \geq 6.5\right\} \\
& T_{32}=\left\{x \in \mathbb{R}^{3} \mid x_{3} \geq 10\right\} \\
& T_{34}=\left\{x \in \mathbb{R}^{3} \mid x_{1} \geq 11\right\} \\
A_{1}= & {\left[\begin{array}{ccc}
-2 . & 5 & -4.9 \\
-5 & -2 & 5.1 \\
0 & 0 & -1.9
\end{array}\right], \quad b_{1}=\left[\begin{array}{c}
7.6 \\
7.6 \\
7.6
\end{array}\right] } \\
A_{2}= & {\left[\begin{array}{ccc}
-1.86 & 0.79 & 0.18 \\
-0.79 & -1 . & -3.92 \\
0.18 & 3.92 & -1.04
\end{array}\right], \quad b_{2}=\left[\begin{array}{c}
9.66 \\
22.78 \\
-11.32
\end{array}\right] } \\
A_{3}= & {\left[\begin{array}{ccc}
-0.7 & -7 & 4.7 \\
7 & -0.7 & -9.3 \\
0 & 0 & -3
\end{array}\right], \quad b_{3}=\left[\begin{array}{c}
12.5 \\
-26 \\
9
\end{array}\right] } \\
A_{4}= & {\left[\begin{array}{ccc}
0.1 & -7 & 4.7 \\
7 & 0.1 & -9 \\
0 & 0 & -3
\end{array}\right], \quad b_{4}=\left[\begin{array}{c}
10 \\
-20 \\
10
\end{array}\right] }
\end{aligned}
$$

Let the set of initial states be a regular icosahedron. Now, examining the eigenvalues of the system matrices we choose to compute an invariant cone $\mathcal{Q C}$, paraboloid $\mathcal{P}$, and ellipsoid $\mathcal{E}$ for system 1, 2, and 3, respectively. Observe that the transition set $T_{32}$ will not be reached by any trajectory starting in the given set of initial states, which can be shown by a subset inclusion test. Hence, no cyclic behavior occurs.

In Figure 2 the projection of the invariant set into $\mathbb{R}^{3}$ are shown as wireframes. The switching planes (i.e., the mode initial sets) and trajectories starting at the vertices of the icosahedron are also shown.

To verify that the discrete state 4 will not be reached we have to prove that $\mathcal{E} \cap T_{41}=\emptyset$. In this case this is easily done by visual inspection. However, there are general methods for deciding if intersections of polyhedral and quadratic sets of any dimension are empty or not, see [10]. 


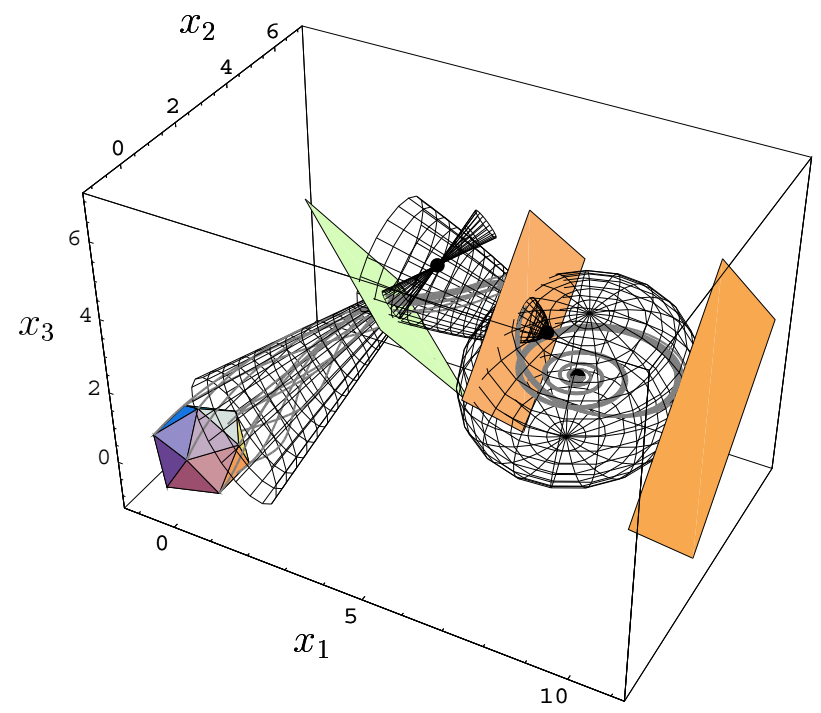

Figure 2: The mode invariant sets, the initial icosahedron in mode 1, switching planes, and a number of trajectories.

Example 2 Consider the hybrid automaton shown in Figure 3.

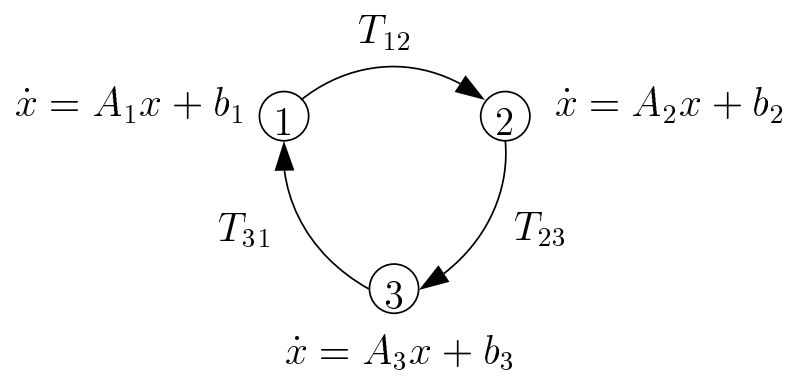

Figure 3: A hybrid automaton of a cycling system.

The three affine systems only differs by a change of coordinates using an orthonormal transformation matrix. The transition sets and affine systems are given by

$$
\begin{aligned}
& T_{12}=\left\{x \in \mathbb{R}^{3} \mid x_{3} \geq 2.5\right\} \\
& T_{23}=\left\{x \in \mathbb{R}^{3} \mid x_{1} \geq 2.5\right\} \\
& T_{34}=\left\{x \in \mathbb{R}^{3} \mid x_{1} \leq 0.5\right\}
\end{aligned}
$$




$$
\begin{aligned}
& A_{1}=\left[\begin{array}{ccc}
-1.96 & -4.9 & 0.09 \\
4.9 & -2 & -1.01 \\
0.29 & 0.97 & -1.04
\end{array}\right], \quad b_{1}=\left[\begin{array}{c}
-0.28 \\
3.02 \\
3.12
\end{array}\right] \\
& A_{2}=\left[\begin{array}{ccc}
-1.5 & -3.54 & -0.5 \\
3.54 & -2 & 3.54 \\
-0.5 & -3.54 & -1.5
\end{array}\right], \quad b_{2}=\left[\begin{array}{c}
4.5 \\
-10.61 \\
1.5
\end{array}\right] \\
& A_{3}=\left[\begin{array}{ccc}
-1.02 & 0.6 & 0.4 \\
-0.4 & -1.99 & -4.96 \\
-0.6 & 4.94 & -1.99
\end{array}\right], \quad b_{3}=\left[\begin{array}{l}
0 \\
0 \\
0
\end{array}\right] .
\end{aligned}
$$

The eigenvalues of all three systems are $-2 \pm 5 i$ and -1 which means that there exists invariant quadratic cones for each system with vertices at the stationary points

$$
x_{01}=\left[\begin{array}{l}
0 \\
0 \\
3
\end{array}\right], \quad x_{02}=\left[\begin{array}{l}
3 \\
0 \\
0
\end{array}\right], \quad x_{03}=\left[\begin{array}{l}
0 \\
0 \\
0
\end{array}\right] \text {. }
$$

Suppose that the initial set is a sphere in mode 1 centered at $(-1.5,0,0)^{T}$ with radius 0.3 . Using the procedure described in Section 4 we get an invariant set of the system after 7 iterations. In Figure 4 we show the projection of the invariant set into $\mathbb{R}^{3}$ as a wireframe, the intersections with the transition set boundaries, and a number of trajectories starting in the initial sphere.

\section{Conclusions}

In this paper we have shown how to compute invariant sets of affine dynamic systems using convex optimization techniques. Utilizing these sets we have proposed a method to compute invariant sets for piecewise affine systems. Invariant sets for this kind of hybrid systems plays an important role in e.g., verification of system properties such as safety, magnitude constraints, etc. Since convex optimization can be performed very efficiently the computations in this paper are scalable to much larger problems.

It should be possible to extend the results taking bounded disturbances into account, i.e., compute sets which are invariant even if disturbances are present. This will be a topic of future research. 


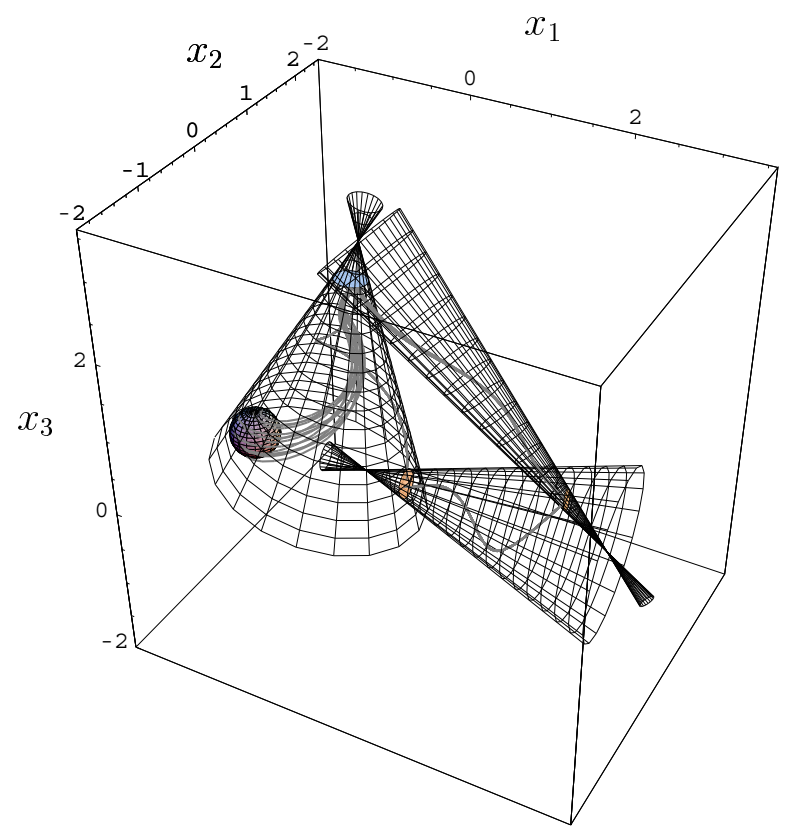

Figure 4: The projection of invariant set into $\mathbb{R}^{3}$ and some trajectories.

\section{Acknowledgment}

This work was supported by the Swedish Research Council for Engineering Sciences (TFR), which is gratefully acknowledged.

\section{References}

[1] R. Alur et al. The algorithmic analysis of hybrid systems. Theoretical Comp. Science, 138, 1995.

[2] K. J. Åström and B. Wittenmark. Computer-Controlled Systems : Theory and Design. Prentice Hall, 1997.

[3] S. Boyd et al. Linear Matrix Inequalities in System and Control Theory. SIAM, 1994.

[4] M. Branicky. Stability of switched hybrid systems. In Proc. of the 33rd IEEE Conf. on Decision and Control, Lake Buena Vista, FL, December 1994. 
[5] L. Breiman. Hinging hyperplanes for regression, classification and function approximation. IEEE Trans. on Information Theory, 39(3), May 1993.

[6] A. Deshpande and P. Varaiya. Viable control of hybrid systems. In Hybrid Systems II, Lecture Notes in Comp. Science. Springer-Verlag, 1995.

[7] N. Halbwachs et al. Verification of linear hybrid systems by means of convex approximations. In Proc. of the First Int. Static Analysis Symposium. Springer-Verlag, 1994.

[8] T. A. Henzinger and P.-H. Ho. A note on abstract interpretation strategies for hybrid automata. In Hybrid Systems II, Lecture Notes in Comp. Science. Springer-Verlag, 1995.

[9] T. A. Henzinger and P.-H. Ho. HyTeCH: The cornell hybrid technology tool. In Hybrid Systems II, Lecture Notes in Comp. Science. SpringerVerlag, 1995.

[10] M. Jirstrand. Constructive Methods for Inequality Constraints in Control. PhD thesis, Department of Electrical Engineering, Linköping University, Sweden, May 1998.

[11] M. Johansson and A. Rantzer. Computation of piecewise quadratic lyapunov functions for hybrid systems. IEEE Trans. on Automatic Control, April 1998.

[12] Mikael Johansson and Anders Rantzer. On the computation of piecewise quadratic lyapunov functions. In Proc. of the 36th IEEE Conf. on Decision and Control, San Diego, CA, December 1997.

[13] H. K. Khalil. Nonlinear Systems. Prentice-Hall, Upper Saddle River, NJ, 2nd edition, 1996.

[14] S. Pettersson and B. Lennartson. Stability and robustness for hybrid systems. In Proc. of the 35th IEEE Conf. on Decision and Control, Kobe, Japan, December 1996.

[15] A. Puri and P. Varaiya. Verification of hybrid systems using abstractions. In Hybrid Systems II, Lecture Notes in Comp. Science. SpringerVerlag, 1995. 
[16] J. Stiver et al. Digital control from a hybrid perspective. In Proc. of the 33rd Conf. on Decision and Control, pages 4241-4246, Lake Buena Vista, FL, December 1994.

[17] J. Stiver et al. Interface and controller design for hybrid systems. In Hybrid Systems II, Lecture Notes in Comp. Science. Springer-Verlag, 1995.

[18] J. A. Stiver et al. An invariant based approach to the design of hybrid control systems containing clocks. In Hybrid Systems III - Verification and Control, Lecture Notes in Comp. Science. Springer-Verlag, 1996.

[19] L. Vandenberghe, S. Boyd, and S.-P. Wu. Determinant maximization with linear matrix inequality constraints. SIAM Journal on Matrix Analysis and Applications, April 1998. To appear.

[20] S.-P. Wu and S. Boyd. Design and implementation of a parser/solver for sdps with matrix structure. In Proc. of IEEE Conf. on Computer Aided Control System Design, 1996.

[21] S.-P. Wu and S. Boyd. sdpsol: Parser/solver for semidefinite programs with matrix structure. In L. El Ghaoui and S.-I. Niculescu, editors, Recent Advances in LMI Methods for Control. SIAM, 1998. To appear. 